\title{
Rehabilitation Needs of Patients with Covid-19 in Acute Phase
}

\author{
Marina Garofano $^{1 *}$, Matteo Maria Ascoli ${ }^{1}$, Roberta Palumbo $^{2}$ and Mariaconsiglia Calabrese ${ }^{3}$ \\ ${ }^{1} P T$, MSc student - Società Italiana Fisioterapia e Riabilitazione (S.I.Fi.R.), Italy \\ ${ }^{2} P T, M S c$ - Società Italiana Fisioterapia e Riabilitazione (S.I.Fi.R.), Italy
}

${ }^{3} P T, P h D$ - President Società Italiana Fisioterapia e Riabilitazione (S.I.Fi.R.), Italy; Physiotherapy Degree Course, Departmentof Medicine, Surgery and Dentistry, University of Salerno, S.Allende Street, 81, 84081 Baronissi, Italy

*Corresponding author: Marina Garofano, Società Italiana Fisioterapia e Riabilitazione (S.I.Fi.R.), Via Alessandro Rossi 114, 80056 Ercolano (NA), Italy

\begin{abstract}
Background: COVID-19 (Coronavirus Disease 19) is a viral infection caused by SARS-Cov-2, that primarily affects the lower respiratory tract and causes several symptoms described as flu-like. Treatment of the disease generally consists of managing clinical symptoms and in case of more severe complications, patients require to be transfer to Intensive Care Units (ICU). Since this type of infection is extremely recent, there are only few studies on the rehabilitation of patients with COVID-19.

Materials and Methods: Papers were identified via a search of PubMed, Scopus and Pedro databases, in order to analyze the role of the physiotherapist in the intensive care units, in the management of non-invasive ventilation (NIV), not forgetting the risk of infection for healthcare professionals.

Conclusion: The analysis of literature carried out shows the importance of the physiotherapists in the management of critically ill patients in the ICU. Key goal of physiotherapy in ICU is the quality of long-term recovery, rather than short-term survival, and physiotherapists play a valuable role in achieving this goal. Finally, in the case of airway infections, the use of Personal Protective Equipment (PPE) should not be overlooked, especially because physiotherapeutic procedures generating aerosols are at high risk of transmission for infections.

Keywords: Physiotherapy; Physiotherapist; Covid-19; Coronavirus; Pulmonary rehabilitation; Chest physiotherapy; Intensive care unit

Abbreviation: ACBT: Active Cycle of Breathing Techniques; BIPAP: Biphasic Positive Airway Pressure; CPAP: Continuous Positive Airway Pressure; ECMO: Extra Corporeal Membrane Oxygenation; ICU: Intensive Care Unit; ILD: Interstitial lung diseases; MERS: Middle East respiratory syndrome; NIV: Non Invasive Ventilation; PPE: Personal Protective Equipment; PEP: Positive Expiratory Pressure; RICU: Respiratory Intensive Care Units; SARS: Severe Acute Respiratory Syndrome; MHI: Manual hyperinflation; PICS: Post-Intensive Care Syndrome
\end{abstract}

\section{Introduction}

COVID-19 (Coronavirus Disease 19) is a viral infection caused by SARS-Cov-2 [1,2], that primarily affects the lower respiratory tract and causes a number of symptoms described as flu-like, including fever, cough, shortness of breath, myalgia and fatigue [1]; in severe cases pneumonia, Acute Respiratory Distress Syndrome (ARDS), sepsis and septic shock can occur, up to the death of the patients [3]. Treatment of the disease generally consists of managing clinical symptoms and in case of more severe complications, such as ARDS, which develops in more than $40 \%$ of hospitalized patients, $10 \%$ require transfer to ICU (Intensive Care Unit) [4].

Since this type of infection is extremely recent, there are only few studies on the rehabilitation of patients with COVID-19, and among them we mention recommendations [5] published in China, a very recent international document [6], and an Italian position paper [7]. This article is a narrative literature review and the papers were identified via a search of PubMed, Scopus and Pedro 
databases, in order to analyze the role of the physiotherapists in intensive care, in the management of NIV (Non Invasive Ventilation) and in relation to infection risk during aerosol-generating procedures. Due to poor results about Sars-Cov-2, we so referred to indications relating to Severe Acute Respiratory Syndrome, SARS (2002), Middle East respiratory syndrome, MERS (2012), Influentia epidemic management.

\section{Literature Analysis}

\section{Role of the physiotherapist in the management of the patient in the ICU}

Faverio et al. [8], in their review, stress the importance of rehabilitation in interstitial lung diseases (ILD) management. ILD are heterogeneous group of diseases characterized by fibrotic and inflammatory anomalies of the lung, so the lung injury and repair caused by COVID-19 has many similarities with ILD [9]. Faverio et al. in agreement with a previous paper [10], compare the efficacy of Extra Corporeal Membrane Oxygenation (ECMO) to invasive ventilation in the treatment of patients with ARDS. The studies highlight the possibility offered by ECMO of early mobilization and walking compared to invasive ventilation; 35 patients of 100 recruited in the study, underwent to physiotherapy, which is not only safe, but also useful for reducing complications and promoting faster recovery $[8,10]$.

In a complete review, all possible interventions of the physiotherapist in Respiratory Intensive Care Units (RICU) are analyzed [11]. Patient management in ICU, includes bed rest still today, especially for patients with mechanical ventilation [12], however, it is already known that rehabilitation is an integral part of patient's management in this setting. Physiotherapy has the following objectives in this context: to improve the patient's functional capacity, to restore respiratory autonomy, and exercise capacity, reducing, therefore, the risks of complications associated with bed rest [13].

The main objective is certainly early weaning from invasive ventilation and many studies have analyzed the effectiveness of physiotherapy intervention in achieving that goal. We will, then, analyze below rehabilitation interventions that can be carried out in RICU, in relation to the effectiveness of each intervention.

\section{Positioning}

It is considered a real treatment. It is used with the aim of improving the ventilation/perfusion ratio (V0/Q0), lung volumes and mucociliary function, to reduce respiratory and cardiac work [13]. Specific examples of positioning to be used in intensive care are: positioning in a semi-upright position, to improve lung volumes and reduce respiratory work in patients who are weaned from mechanical ventilation; prone position to improve the ventilation/ perfusion ratio, redistribute edema and increase residual functional capacity (short-term oxygenation improvements for $57-92 \%$ of patients with severe acute respiratory failure)[13-15]. Regarding prone positioning, a strong appreciation is addressed to physiotherapist's positioning management due to the low incidence of accidental extubations, hypotensive crises and arrhythmia [14].

\section{Active/passive mobilization}

Have significant effects on functional status, muscle strength, duration of mechanical ventilation, ability to walk and quality of life. Early mobilization is associated with greater exercise capacity at discharge, with an increase in the functional capacity of muscle strength $[17,18]$. There are some evidence also in favor of the use of the cycle ergometer and electrical neuromuscular stimulation [19]. Revisions about the use of neuromuscular stimulation, however, report very low- or low-quality tests on the beneficial effects of electrical muscle stimulation provided in ICU to improve muscle strength, trophism and polyneuropathy/myopathy [20,21].

\section{Chest rehabilitation}

Includes early mobilization, inspiratory muscle training, manual hyperinflation, percussion/vibration of the chest wall, removal of secretions, guided cough. Study's results indicate that chest physiotherapy in ICU reduces extubation failure in patients with mechanical ventilation and significantly improves respiratory parameters, reduce the pulmonary infection rate, length of stay on mechanical ventilation, hospitalization and mortality [22-24].

\section{Manual hyperinflation (MHI)}

Maneuver frequently used in seriously ill patients intubated and mechanically ventilated. Patients are disconnected from the mechanical ventilator and after their lungs are temporarily ventilated with a balloon for manual ventilation usually with the addition of $100 \%$ oxygen [25]. This maneuver is useful to simulate a physiological and effective cough. By moving the secretions of the smaller airways to the larger ones, it is then possible to easily remove the secretions by aspiration. In Paulus et al.'s review [26] there are no indications for the use of this technique in unstable, intubated and mechanically ventilated patients. Manual hyperinflation does not appear to be associated with a reduction in the duration of mechanical ventilation, permanence in the ICU and prevention of ventilator-associated pneumonia [26], and further studies are necessary to investigate the use of MHI by physical therapists for better understanding benefits and intrinsic risks [27]. Very interesting is a review investigating the role of physiotherapy in reducing the symptoms of post-intensive care syndrome (PICS) [28]. This syndrome is characterized by psychophysical problems that occur in critically ill survivors of ICU [29]; these impairments can be long lasting and adversely affect self-perceived quality-of-life. The potential benefits for patients participating in early rehabilitation in ICU are improvement of muscle strength, quality of life, reduction of hospital and intensive 
care stay, reduction of mechanical ventilation and costs [30-32]. The studies analyzed show the importance of the multidisciplinary team in management of the critically ill patient and the need for evidence-based approaches for which research should certainly be implemented [28].

\section{Role of the physiotherapist in the management of Non- Invasive Ventilation (NIV)}

Non-invasive ventilation (NIV) is a ventilation technique that provide ventilatory support for a patient with respiratory problems without a need for endotracheal intubation or tracheostomy. Understanding how the technique works and how to apply it appropriately is necessary to manage patients with respiratory failure effectively and appropriately [33]. Physiotherapists with advanced or specialized respiratory skills are well integrated in the assistance and management of patients who use NIV, also integrating airway clearance techniques such as respiratory exercises, use of Positive Expiratory Pressure (PEP), active cycle of breathing techniques (ACBT), assisted cough to manage acute and chronic respiratory failure [33]. A recent review aimed at producing guidelines on the training of healthcare professionals working with NIV. No clinical studies that examine the impact of education and training in the use of NIV as a primary objective were found. Therefore, some studies with indirect evidence have been identified, including a study based on simulation and narrative reviews. The review concludes that education and training have the potential to increase the knowledge and skills of staff using NIV. It is therefore desirable that all health professionals, and among them physiotherapists, participate in dedicated and homogeneous training programs at international level [34].

Finally, we analyzed a Italian study in which 201 patients were recruited in order to define a standardized protocol of adaptation to NIV, for patients with chronic respiratory diseases and, in the second instance, evaluate the role of the physiotherapist, also in terms of time used for each patient to educate him on the use of NIV [35]. As emerged from previous studies, the role of the physiotherapists in the United States is different Italy due to education system, and in usually focused on the choice of the ventilator and the mask, on the setting of the ventilator and on patient's training, while the role of the doctor is mainly related to the management of NIV [36-38]. Nursing staff are usually identified as being responsible for night monitoring and side effects such as damage to facial skin [36-38]. In Italy there are some differences in educations terms compared to the United States, and therefore also the activities carried out by physiotherapists with advanced skills and specific education in the respiratory field are different. In this study it emerges that they can play a key role in the management of patients with chronic respiratory diseases, sleep disorders, and with neuromuscular diseases [35]. The inclusion of physiotherapists in the management of NIV and the standardization of procedures seems to reduce the time spent by other healthcare professionals in patient education by optimizing the management of the activities carried out. The cost-effectiveness of this management must be verified in wider clinical trial contexts.

\section{Procedures at risk of infection for the physiotherapist}

In rehabilitation treatment of patients with Covid-19 in acute phase, the risk of infection for physiotherapists must be taken into great consideration since the rehabilitation techniques used normally are responsible for the generation of aerosols. The Canadian Guidelines developed in 2011 [39] on the occasion of the SARS Coronavirus epidemic, report the treatments most closely linked to the risk of infection for healthcare professionals: Biphasic Positive Airway Pressure (BIPAP), Continuous Positive Airway Pressure (CPAP), endotracheal intubation, airway aspiration, high frequency oscillatory ventilation, tracheostomy, chest physiotherapy, nebulizer treatments, airway clearance methods and bronchoscopy [40]. From the afore mentioned Guidelines [38] and from the studies conducted on the occasion of the SARS-Coronavirus epidemic, it appears evident the risk for physiotherapists who deal with acute respiratory rehabilitation for infected patients [40-42]. A risk reduction can be obtained with the use of Personal Protective Equipment (PPE), in particular a N95/ FFP3 mask or equivalent, a completely waterproof gown, a visor, goggles and gloves, moreover these procedures should be carried out in a single room with a minimum number of highly qualified healthcare professionals present and especially in case of need $[7,40,43]$.

\section{Conclusion}

The analysis of literature carried out shows the importance of the physiotherapists in the management of critically ill patients in ICU. The ICU is a dynamic environment in which physiotherapists are an integral part of the multidisciplinary team of critical area providing various types of interventions, from chest physiotherapy in the acute phase, to prevention and recovery of motor deficits. Key goal of physiotherapy in ICU is the quality of long-term recovery, rather than short-term survival, and physiotherapists play a valuable role in achieving this goal [44]. It also Appears also to be important to define educational strategies both for healthcare professionals, so that they are properly educates, and for patients, who must be educated in the use of the devices, trying if possible, to produce shared guidelines $[17,18]$. Finally, in the case of airway infections, the use of PPE should not be overlooked [45], as the physiotherapeutic procedures generating aerosols are considered to be at high risk of transmission for infections as already seen for SARS-Coronavirus $2[39,40]$ and therefore potentially dangerous for the physiotherapists who use them. 


\section{Acknowledgements}

All authors contributed to the literature search, identification of relevant studies, and writing of the paper.

\section{Conflict of Interest}

The authors declare no conflict of interest.

\section{References}

1. Gu J, Han B, Wang J (2020) COVID-19: Gastrointestinal manifestations and potential fecal-oral transmission. Gastroenterology.

2. Guo YR, Cao QD, Hong ZS, Tan YY, Chen SD, et al. (2020) The origin, transmission and clinical therapies on coronavirus disease 2019 (COVID-19) outbreak-an update on the status. Military Medical Research 7(1): 1-10.

3. Velavan TP, Meyer CG (2020) The COVID-19 epidemic. Tropical Medicine and International Health 25(3): 278-280.

4. Wu C, Chen X, Cai Y, Xia J, Zhou X, et al. (2020) Risk Factors Associated with Acute Respiratory Distress Syndrome and Death in Patients with Coronavirus Disease 2019 Pneumonia in Wuhan, China. JAMA Internal Medicine 1-10.

5. (2020) Chinese Association of Rehabilitation Medicine; Respiratory rehabilitation committee of Chinese Association of Rehabilitation Medicine; Cardiopulmonary rehabilitation Group of Chinese Society of Physicai Medicine and Rehabilitation. [Recommendations for respiratory rehabilitation of COVID-19 in adult] Zhonghua Jie He He Hu Xi Za Zhi 40(0).

6. Thomas P, Baldwin C, Bissett B, Boden I, Gosselink R, et al. (2020) Physiotherapy management for COVID-19 in the acute hospital setting. Recommendations to guide clinical practice. Journal of Physiotherapy.

7. Lazzeri M, Lanza A, Bellini R, Bellofiore A, Cecchetto S, et al. (2020) Respiratory physiotherapy in patients with COVID-19 infection in acute setting: A Position Paper of the Italian Association of Respiratory Physiotherapists (ARIR). Monaldi Archives for Chest Disease, 90(1).

8. Faverio P, De Giacomi F, Sardella L, Fiorentino G, Carone M, et al. (2018) Management of acute respiratory failure in interstitial lung diseases: Overview and clinical insights. BMC Pulmonary Medicine 18(1): 1-13.

9. Ren YH, Wang SY, Liu M, Guo YM, Dai HP [When COVID-19 encounters interstitial lung disease: challenges and management]. Zhonghua Jie He He Hu Xi Za Zhi 43(0).

10. Abrams D, Javidfar J, Farrand E, Mongero LB, Agerstrand CL, et al. (2014). Early mobilization of patients receiving extracorporeal membrane oxygenation: A retrospective cohort study. Critical Care 18(1): 1-9.

11. Clini E, Ambrosino N (2005) Early physiotherapy in the respiratory intensive care unit. Respiratory Medicine 99(9): 1096-1104.

12. Needham DM (2008) Mobilizing patients in the intensive care unit: improving neuromuscular weakness and physical functionitle. JAMA Internal Medicine 300:1685-1.

13. Parker AM, Sricharoenchai T, Needham DM (2013) Early Rehabilitation in the Intensive Care Unit: Preventing Impairment of Physical and Mental Health. Current Physical Medicine and Rehabilitation Reports 1(4): 307-314.

14. Cristina K, Paiva DEA, Beppu OS (2005) Prone position. 332-340.

15. Kallet RH (2015) A comprehensive review of prone position in ARDS. Respiratory Care 60(11):1660-1687.

16. Koulouras V, Papathanakos G, Papathanasiou A, Nakos G (2016) Efficacy of prone position in acute respiratory distress syndrome patients: A pathophysiology-based review. World Journal of Critical Care Medicine 5(2):121
17. Arias-Fernández P, Romero-Martin M, Gómez-Salgado J, FernándezGarcía D (2018) Rehabilitation and early mobilization in the critical patient: systematic review. Journal of Physical Therapy Science 30(9): 1193-1201.

18. Stiller K (2013) Physiotherapy in intensive care: An updated systematic review. Chest 144(3): 825-847.

19. Connolly B, O'Neill B, Salisbury L, Blackwood B (2016) Physical rehabilitation interventions for adult patients during critical illness: An overview of systematic reviews. Thorax 71(10): 881-890.

20. Hermans G, De Jonghe B, Bruyninckx F, Van den Berghe G (2014) Interventions for preventing critical illness polyneuropathy and critical illness myopathy. Cochrane Database of Systematic Reviews 2014(1).

21. Wageck B, Nunes GS, Silva FL, Damasceno MC (2014) Application and effects of neuromuscular electrical stimulation in critically ill patients: systematic review. Med Intensiva.

22. Castro AAM, Calil SR, Freitas SA, Oliveira AB, Porto EF (2013) Chest physiotherapy effectiveness to reduce hospitalization and mechanical ventilation length of stay, pulmonary infection rate and mortality in ICU patients. Respiratory Medicine 107(1): 68-74.

23. Denehy L, Berney S (2006) Physiotherapy in the intensive care unit. Physical Therapy Reviews 11(1): 49-56.

24. Wang TH, Wu CP, Wang LY (2018) Chest physiotherapy with early mobilization may improve extubation outcome in critically ill patients in the intensive care units. Clinical Respiratory Journal 12(11): 2613-2621.

25. Denehy L (1999) The use of manual hyperinflation in airway clearance. European Respiratory Journal 14(4): 958-965.

26. Paulus F, Binnekade JM, Vroom MB, Schultz MJ (2012) Benefits and risks of manual hyperinflation in intubated and mechanically ventilated intensive care unit patients: a systematic review. Critical Care 16(4): R145.

27. Cruz RVS, do Socorro da Silva Dias de Andrade F, Menezes PD, Gonçalves BO, Almeida R da S, Santos A R (2017). Manual hyperinflation and the role of physical therapy in intensive care and emergency units TT- 241248.

28. Parker AM, Sricharoenchai T, Needham DM (2013) Early Rehabilitation in the Intensive Care Unit: Preventing Impairment of Physical and Mental Health. Current Physical Medicine and Rehabilitation Reports 1(4): 307-314.

29. Needham D, Davidson J, Cohen H, Hopkins RO, Weinert C, et al. (2012) Improving long-term outcomes after discharge from intensive care unit: report from a stakeholders' conference. Crit Care Med 40(2): 502-509.

30. Adler J, Malone D (2012) Early mobilization in the intensive care unit: a systematic review. Cardiopulm Phys Ther J 23(1): 5-13.

31. Needham DM (2008) Mobilizing patients in the intensive care unit: improving neuromuscular weakness and physical function. JAMA 300(14): 1685-16851.

32. Li Z, Peng X, Zhu B, Zhang Y, Xi X (2013) Active mobilization for mechanically ventilated patients: A systematic review. Archives of Physical Medicine and Rehabilitation, 94(3): 551-561.

33. Piper AJ, Moran FM (2006) Non-invasive ventilation and the physiotherapist: current state and future trends. Physical Therapy Reviews 11(1): 37-43.

34. Reazaul Karim HM, Burns KEA, Ciobanu LD, El-Khatib M, Nicolini A, et al. (2019) Noninvasive ventilation: Education and training. A narrative analysis and an international consensus document. Advances in Respiratory Medicine 87(1): 36-45.

35. Simonelli C, Paneroni M, Vitacca M (2013) An implementation protocol for noninvasive ventilation prescription: The physiotherapist's role in an Italian hospital. Respiratory Care 58(4): 662-668. 
36. Hess DR (2009) How to Initiate a Noninvasive Ventilation Program: Bringing the Evidence to the Bedside. Respir Care 54(2): 232-243.

37. Harris C, Saskin R, Burns KE (2010) Noninvasive Ventilation Initiation in Clinical Practice: A Six-Year Prospective, Observational Study. Canadian Respiratory Journal 17(3): 123-131.

38. Davies JD, Gentile MA (2009) What does it take to have a successful noninvasive ventilation program? Respiratory Care 54(1): 53-61.

39. Tran K, Cimon K, Severn M, Pessoa-Silva CL, Conly J (2012) Aerosol generating procedures and risk of transmission of acute respiratory infections to healthcare workers: A systematic review. PLoS ONE 7(4).

40. Lim WS, Anderson SR, Read RC (2004) Hospital management of adults with severe acute respiratory syndrome (SARS) if SARS re-emergesUpdated 10 February 2004. Journal of Infection 49(1):1-7.

41. Public Health Ontario (2015) Infection Prevention and Control for Clinical Office Practice. Queen's Printer for Ontario 114.
42. Siegel JD, Rhinehart E, Jackson M, Chiarello L (2009) Guideline for isolation precautions: Preventing transmission of infectious agents in healthcare settings 2007. Hospital-Acquired Infections 1-232.

43. WHO (2020) Global surveillance for COVID-19 disease caused by human infection with the 2019 novel corona virus. Interim guidance. February 2020. Available from: https://www.who.int/publications-detail/globalsurveillance-for-human-infection-with-novel-coronavirus-(2019-ncov)

44. Pathmanathan N, Beaumont N, Gratrix A (2015) Respiratory physiotherapy in the critical care unit. Continuing Education in Anaesthesia, Critical Care and Pain 15(1): 20-25.

45. Report ET (2020) Infection prevention and control for COVID-19 in healthcare settings Target audience Healthcare settings.
(C) (P) This work is licensed under Creative

To Submit Your Article Click Here:

Submit Article

DOI: 10.32474/RRHOAJ.2020.05.000204

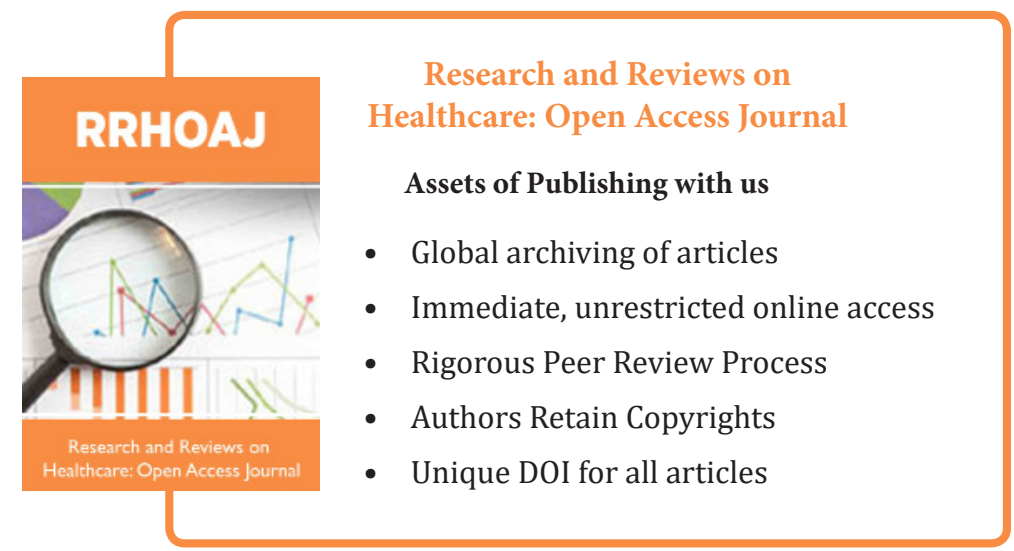

